\title{
Clinical Study End Date
}

National Cancer Institute

\section{Source}

National Cancer Institute. Clinical Study End Date. NCI Thesaurus. Code C90462.

The date on which the study concludes. 